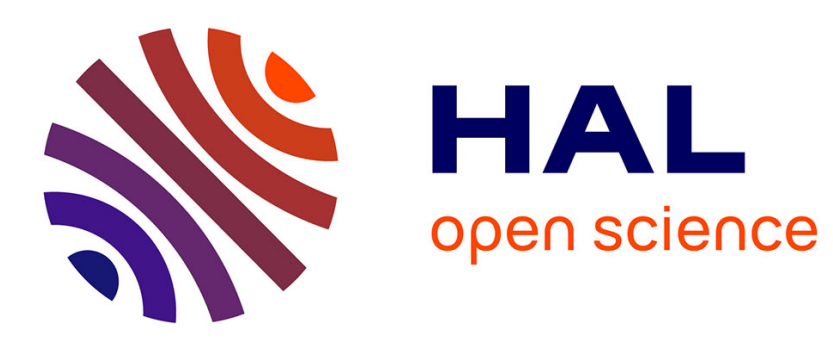

\title{
Solutions for Processing K Nearest Neighbor Joins for Massive Data on MapReduce
}

\author{
Ge Song, Justine Rochas, Fabrice Huet, Frédéric Magoulès
}

\section{To cite this version:}

Ge Song, Justine Rochas, Fabrice Huet, Frédéric Magoulès. Solutions for Processing K Nearest Neighbor Joins for Massive Data on MapReduce. 23rd Euromicro International Conference on Parallel, Distributed and Network-based Processing, Mar 2015, Turku, Finland. hal-01097337

\section{HAL Id: hal-01097337 https://hal.inria.fr/hal-01097337}

Submitted on 12 Jan 2015

HAL is a multi-disciplinary open access archive for the deposit and dissemination of scientific research documents, whether they are published or not. The documents may come from teaching and research institutions in France or abroad, or from public or private research centers.
L'archive ouverte pluridisciplinaire HAL, est destinée au dépôt et à la diffusion de documents scientifiques de niveau recherche, publiés ou non, émanant des établissements d'enseignement et de recherche français ou étrangers, des laboratoires publics ou privés. 


\title{
Solutions for Processing K Nearest Neighbor Joins for Massive Data on MapReduce
}

\author{
Ge Song*†, Justine Rochas*, Fabrice Huet* and Frédéric Magoulès ${ }^{\dagger}$ \\ ${ }^{*}$ Univ. Nice Sophia Antipolis, CNRS, I3S, UMR 7271, 06900 Sophia Antipolis, France \\ ${ }^{\dagger}$ Ecole Centrale de Paris, France \\ ge.song@inria.fr, \{justine.rochas,fabrice.huet\}@unice.fr, frederic.magoules@ecp.fr
}

\begin{abstract}
Given a point $p$ and a set of points $S$, the kNN operation finds the $k$ closest points to $p$ in $S$. It is a computational intensive task with a large range of applications such as knowledge discovery or data mining. However, as the volume and the dimension of data increase, only distributed approaches can perform such costly operation in a reasonable time. Recent works have focused on implementing efficient solutions using the MapReduce programming model because it is suitable for large scale data processing. Also, it can easily be executed in a distributed environment. Although these works provide different solutions to the same problem, each one has particular constraints and properties. There is no readily available comparison to help users choose the one most appropriate for their needs. This is the problem we address in this work. Firstly, we show that all kNN implementations go through a common workflow, which we use as a basis for classification. Secondly, we describe precisely the different techniques published so far. And lastly, we provide a set of objective criteria that can be used to make informed decisions.
\end{abstract}

Keywords—kNN Join; Data Partition; Hadoop; MapReduce;

\section{INTRODUCTION}

Given a set of query points $R$ and a set of reference points $S$, a $k$ nearest neighbor join (hereafter $\mathrm{kNN}$ ) is an operation which, for each point in $R$, discovers the $k$ nearest neighbors in $S$. It is frequently used as a classification or clustering method in machine learning or data mining. It can be applied to a large number of fields, such as multimedia [1] [2], social network [3], time series analysis [4] [5], bio-information and medical imagery [6] [7].

The basic idea to compute a $\mathrm{kNN}$ is to perform a pairwise computation of distance for each element in $R$ and each element in $S$. The computational complexity of this pairwise calculation is $O(|R| \times|S|)$. Then, finding the $k$ nearest neighbors in $S$ for every $r$ in $R$ boils down to sorting the computed distances, and leads to a complexity of at least $|S| \times \log |S|$. As the amount of data or their complexity (number of dimensions) increases, this approach becomes impractical. This is why a lot of work has been dedicated to reducing the $\mathrm{kNN}$ computational complexity [8] [9] [10] [11] [12].

Although a variety of previous work has greatly improved its performance, there are still significant limitations to process $\mathrm{kNN}$ on a single machine when the amount of data increases. For large dataset, only distributed and parallel solutions prove to be powerful enough. MapReduce is a flexible and scalable parallel and distributed programming paradigm which is especially designed for data-intensive processing. It was first introduced by Google [13] and popularized with the Hadoop [14] framework, an open source implementation. MapReduce offers a programming model adapted to large scale data processing, which can easily be distributed. The framework can be installed on commodity hardware and automatically distribute a MapReduce job over a set of machines. Therefore, it seems to be a good target for computing $\mathrm{kNN}$ in a distributed way. However, writing an efficient $\mathrm{kNN}$ in MapReduce is challenging. First, classical algorithms have to be redesigned to fit the MapReduce programming model. Second, data partition and distribution strategies have to be carefully designed to limit communications and data transfer. In this paper, we review existing implementations of $\mathrm{kNN}$ in MapReduce, focusing on different steps involved in a computation. Other surveys about $\mathrm{kNN}$ have been conducted, such as [15], [16]. In [15], the authors only focus on centralized solutions to optimize kNN computation whereas we target distributed solutions. In [16], the survey is also oriented towards centralized techniques and is based on a theoretical performance analysis. Our approach is much more high level, though we also provide some details about the performance of the works we study. Overall, our contributions are:

- The decomposition of a distributed $\mathrm{kNN}$ computation in different steps.

- An analysis of existing techniques for each step based on load balancing, accuracy and complexity.

- A global comparison which can be used to find the most suitable solution for a given use case.

The rest of the paper is organized as follows. Section II introduces the background about $\mathrm{kNN}$ and MapReduce. Section III presents the 3 stages of workflow involved in computing kNN on MapReduce. Section IV analyzes existing implementations from the following points of view: load balancing, accuracy and complexity. Finally, Section V concludes this work.

\section{CONTEXT}

\section{A. $k$ Nearest Neighbors}

A nearest neighbors query consists in finding at most $k$ points in a data set $S$ that are the closest to a considered point $r$, in a dimensional space $d$. More formal definitions are as follows: given two data sets $R$ and $S$ in $\mathbf{R}^{d}$, and given $r$ and $s$, two elements, with $r \in R$ and $s \in S$, we have:

Definition 1: Let $d(r, s)$ be the distance between $r$ and $s$. The kNN query of $r$ over $S$, noted $k N N(r, S)$ is the subset $\left\{s_{i}\right\} \subseteq S\left(\left|\left\{s_{i}\right\}\right|=k\right)$, which is the $k$ nearest neighbors of 
$r$ in $S$, where $\forall s_{i} \in k N N(r, S), \forall s_{j} \in S-k N N(r, S)$, $d\left(r, s_{i}\right) \leq d\left(r, s_{j}\right)$.

It is easy to extend the previous definition to a set of query points.

Definition 2: The kNN join of two datasets $R$ and $S$, $\operatorname{kNN}(R \ltimes S)$ is:

$$
\operatorname{kNN}(R \ltimes S)=\{(r, \operatorname{kNN}(r, S)), \forall r \in R\}
$$

Depending on the use case, it might not be necessary to find the exact solution of a kNN query, and that is why approximate $\mathrm{kNN}$ queries have been introduced. The idea is to have the $k^{t h}$ approximate neighbor not far from the $k^{\text {th }}$ exact one, as shown in the following definition.

Definition 3: The $(1+\epsilon)$-approximate kNN query for a query point $r$ in a dataset $S, A k N N(r, S)$ is a set of approximate $k$ nearest neighbors of $r$ from $S$, if the $k^{\text {th }}$ furthest result $s^{k *}$ satisfies $s^{k *} \leq s^{k} \leq(1+\epsilon) s^{k *}(\epsilon>0)$ where $s^{k}$ is the exact $k^{\text {th }}$ nearest neighbor of $r$ in $S$.

And as with exact $\mathrm{kNN}$, this definition can be extended to an approximate $\mathrm{kNN}$ join.

Definition 4: The $(1+\epsilon)$-approximate kNN join of two datasets $R$ and $S, \operatorname{AkNN}(R \ltimes S)$ is:

$$
\operatorname{AkNN}(R \ltimes S)=\{(r, \operatorname{AkNN}(r, S)), \forall r \in R\}
$$

The basic solution to compute kNN adopts a block nested loop approach, which calculates the distance between every object $r_{i}$ in $R$ and $s_{j}$ in $S$ and sorts the results to find the $k$ smallest. This approach is computational intensive, making it unpractical for large or intricate datasets. Two strategies have been proposed to work out this issue.

The first one consists in reducing the number of distances to compute, by avoiding scanning the whole dataset. This strategy focuses on indexing the data through efficient data structures. For example, a one-dimension index structure, the $B^{+}$-Tree, is used in [8] to index distances; [9] adopts a multipage overlapping index structure R-Tree, whose minimum bound is a rectangle (MBR); [10] proposes to use a balanced and dynamic M-Tree to organize the dataset; [17] introduces a sphere-tree with a sphere-shaped minimum bound to reduce the number of areas to be searched; [18] presents a multidimensional quadtree in order to be able to handle large amount of data; and [12] develops a kd-tree which is a clipping partition method to separate the search space.

However, reducing the searched dataset might not be sufficient. For data in large dimension space, computing the distance might be very costly. That is why a second strategy focuses on projecting the high-dimension dataset onto a lowdimension one, while maintaining the locality relationship between data. Representative efforts refer to LSH (LocalitySensitive Hashing) [19] and Space Filling Curve [20]. In these cases, the low-dimension data contain much less information than the high-dimension ones because in most cases, the lowdimension data cannot totally fill the high-dimension space. To keep as much information as possible, the trick is to project the high-dimension data multiple times from different perspectives, producing multiple new datasets. For instance, LSH usually provides many space hashing functions. The space filling curve method also usually needs several shifts of data, to reduce the possibilities of errors in the data locality when projecting.

But with the increasing amount of data, these methods can still not handle kNN computation on a single machine efficiently. Experiments in [21] suggest using GPUs to significantly improve the performance of distance computation, but this is still not applicable for large datasets that cannot reasonably be processed on a single machine.

Recent papers have focused on providing efficient distributed implementations. Some of them use ad hoc protocols based on well-known distributed architectures [22], [23]. But most of them use the MapReduce model as it is naturally adapted for distributed computation, like in [24]-[26]. In this paper, we focus on the kNN computing systems based on MapReduce, because such systems can scale with the predictable data growth, and also because the framework on which they are based is widespread.

\section{B. MapReduce}

MapReduce [13] is a parallel programming model that aims at efficiently processing large datasets. This programming model is based on three concepts: (i) representing data as keyvalue pairs, (ii) defining a map function, and (iii) defining a reduce function. The map function takes key-value pairs as an input, and produces zero or more key-value pairs. Outputs with the same key are gathered together (shuffled) so that key-\{list of values $\}$ pairs are given to reducers. The reduce function processes all the values associated with a given key.

This decomposition enables data parallelism as the only dependency between the output of the maps and input of reduces. In practice, the map and reduce functions can be executed on many machines, leading to a naturally distributed computation. The most famous implementation of this model is the Hadoop framework [14] which provides a distributed platform for executing MapReduce jobs.

\section{WORKFLOW}

In this section, we review the methods that have been used to perform kNN join on MapReduce. Overall, they all share the same workflow, comprised of three stages: (i) data preprocessing (ii) data partitioning and organization and (iii) computation of kNN. We will focus on the following works: the initial (basic, not optimized) idea, which we call $\mathrm{H}-\mathrm{BkNNJ}$, and its improvements H-BNLJ (Block Nested Loop Join) and H-BRJ (Block Nested R-Tree Join) in [26], as well as more advanced solutions such as H-zkNNJ (z-value) in [26], RankReduce (Locality Sensitive Hashing) in [24] and PGBJ (Voronoi) in [25].

\section{A. Data Preprocessing}

The preprocessing stage aims either at processing the initial dataset to reduce its complexity or produces extra information about the data. It is an optional step, as some algorithms can directly work on the initial data, as H-BkNNJ, H-BNLJ and H-BRJ [26].

For high-dimensional data, the first pre-processing approach projects data onto low-dimensional ones. One way 
to reduce the complexity and size of the dataset is to use space-filling curves as shown in [20]. The solution consists in mapping high-dimensional data to low-dimensional ones while maintaining the locality relationship between each object with high probability (two elements that are close in a high-dimensional space should remain close in the reduced dimensional space). In [26], the authors compute the $z$-value of all data from $R$ and $S$. The $z$-value of a data is a one dimensional value that is calculated by interleaving the binary representation of the coordinates from MSB (Most Significant Bit) to LSB (Least Significant Bit). However, the ability of $z$-value to maintain the relative locality between objects in space is not good enough, especially when the dimension is high. Therefore, in practice, several random shifts of the data are generated, and additional $z$-values are computed, which aims to improve the accuracy at the cost of computation and space.

Another way to reduce the dimension of data is LSH. In RankReduce [24], high-dimensional data are projected onto low-dimensional ones using Locality Sensitive Hashing (LSH). The idea is to use a group of locality preserving hash functions to map close points from the original data to the same hash value with high probability. The overall performance of LSH depends on parameter tuning [27] which depends on the original dataset. Although it is likely for related objects to have the same hash values, in general, one single hash function cannot guarantee a satisfying accuracy. Often, a group of hash functions is required in order to generate multiple hash tables to reduce the number of collisions for distant objects.

In fact, as the quality of the projection is data dependent, both solutions in [26] and [24] duplicate the initial dataset and use different parameters for the projection to end up with several projected datasets. The purpose of this duplication is to alleviate information loss from the initial dataset: having multiple projected datasets enable to isolate potential errors. Overall, those solutions are willing to pay the same computation several times on different projected datasets in trade for the complexity of data.

Another approach consists in selecting leaders in the dataset which will drive the subsequent computation. In PGBJ [25], the preprocessing phase tries to identify pivot points (points that correspond more or less to the barycenter of a cluster of points) in the initial dataset which will lead a partition of the dataset. Thus, it is a primary selection before the partitioning stage. In paper [25], three strategies to select the pivots are described. The "Random Selection" strategy generates a set of samples, and calculates the pairwise distance for every point in the samples, and then sums all the distances together. Then, the sample with the biggest summation of distances will be chosen as set of pivots. This strategy provides good results as long as the sample sets are big enough, to maximize the chance to select points in different clusters. The "Farthest Selection" strategy randomly chooses the first pivot. Then, the farthest point to the first pivot will be chosen as the second pivot, and so on until having the desired number of pivots. This strategy ensures that the distance between each selected point is as large as possible, but it is heavier to process than the previous one, as it requires the computing of a lot of distances. Finally, the "K-Means Selection" applies the traditional k-means method on a data sample to update the centroid of a cell as the new pivot each step, until the pivots do not change. With this strategy, the pivots are ensured to be in the middle of a cluster of points, but it is the heaviest strategy as it needs to converge towards the optimal solution. As we will see in the next section, the quality of pivots has an important impact on the quality of the partitioning.

\section{B. Data Partitioning and Organization}

First attempts to compute kNN efficiently in sharedmemory focused on particular data organizations, such that the neighbor set can be pruned and neighbor sorting is performed faster. In the most popular methods, data are usually indexed using a tree structure like $B^{+}$-Tree [8] or R-Tree [9]. But as we target big data, shared-memory centric solutions cannot be easily applied to a shared-nothing platform as MapReduce. Instead, the dataset need to be separated into several sets, called partitions, such that, ideally, each partition is independent from others. It is nonetheless possible to use efficient data structures to improve local searching in a partition.

As in any MapReduce computation, the data partition strategy will strongly impact CPU, network communication and disk usage, which in turn will impact the overall processing time [28]. The key to improve the performance is to preserve spatial locality of objects when decomposing data for tasks [29]. This means making a coarse clustering in order to produce a reduced set of neighbors that are candidates for the final result. Intuitively, the goal is to have a partitioning of data such as an element in a partition of $R$ will have its nearest neighbors in only one partition of $S$. More precisely, what we want is:

For every partition $R_{i}\left(\cup_{i} R_{i}=R\right)$, find a corresponding partition $S_{j}\left(\cup_{j} S_{j}=S\right)$, where

$$
\begin{gathered}
k N N\left(R_{i} \ltimes S\right)=k N N\left(R_{i} \ltimes S_{j}\right), \text { and, } \\
k N N(R \ltimes S)=\bigcup k N N\left(R_{i} \ltimes S_{j}\right)
\end{gathered}
$$

which means that, not only it is possible to compute $\mathrm{kNN}$ for each element of $R_{i}$ in a single $S_{j}$, but also the concatenation of the results for all $R_{i}$ is equal to the global $\mathrm{kNN}$ join.

In this section, we present two partition methods that enable to separate the dataset into shared-nothing subsets while preserving locality information.

1) Distance Based Partitioning: The first partitioning method is based on Voronoi diagram, a method to divide the space into disjoint cells. The main property of this method is that every point in a cell is closer to the pivot of this cell than to any other pivot. Because this method relies on the distance metric, it is naturally used to solve neighborhood problems. More formally, the definition of a Voronoi cell is as follow:

Definition 5: Given a set of disjoint pivots: $\mathrm{P}=$ $\left\{p_{1}, p_{2}, \ldots, p_{i}, \ldots, p_{n}\right\}$, the Voronoi Cell of $p_{i}(0<i \leq n)$ is: $\forall \mathrm{i} \neq \mathrm{j}, V C\left(p_{i}\right)=\left\{p \| d\left(p, p_{i}\right) \leq d\left(p, p_{j}\right)\right\}$.

Paper [25] gives a method to partition the dataset $R$ and $S$ using Voronoi diagram. After having identified the pivots in $R$ (as seen in the preprocessing section - III-A), they compute the distance between every point and the pivots, and put the considered point into the cell of the closest pivot. This will naturally give a partitioning of data. Afterwards, they also 


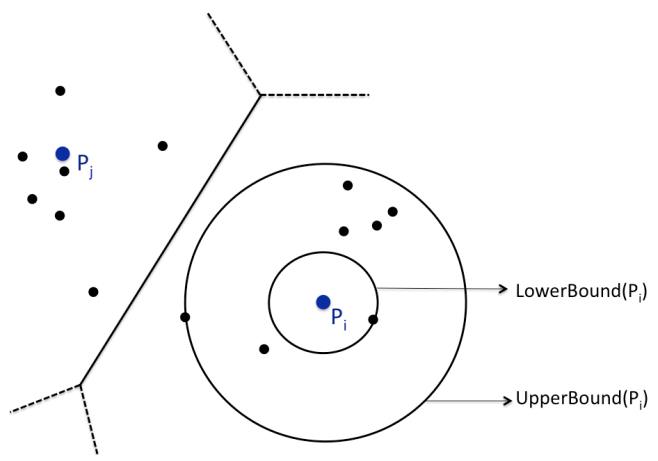

Fig. 1: Voronoi based partitioning for pivots $P_{i}$ and $P_{j}$

calculate, for each cell of $R$, the upper (resp. lower) bound of the partition which corresponds to the sphere determined by the furthest (resp. closest) point (in $S$ ) from the pivot in the cell of $R$. Such bounds enable to quickly find the correct partition $S_{j}$ for a given partition $R_{i}$. This data partitioning principle is shown in Figure 1 where two pivots $P_{i}$ and $P_{j}$ have been chosen from the dataset.

The main problem of this method is that it requires computing the distance of all elements to the pivots. Also, the distribution of the input data might not be known in advance. Hence, the pivots will have to be recomputed if the data changes, which limits dynamicity. Also, there is no guarantee that all cells will have an equal number of elements because of potential data skew. This can have a negative impact on the overall performance because of load balancing issues.

2) Size Based Partitioning: Another type of partitioning method aims at dividing the data into some equal size partitions while preserving their locality information. [26] proposes a partitioning strategy based on $z$-value described in the previous section. In order to make every partition of $R$ have a similar number of objects, a sampling is first performed. They claim that the $n$ quantiles ( $n$ is equal to the number of partitions) of the sampling data is an unbiased estimation of the boundary point of each partition, with a standard deviation $\leq \epsilon|R|(\epsilon>0)$. After having partitioned $R$ this way, the same sampling strategy is applied to $S$. For each of the boundary points of all $R_{i}$, the corresponding $k^{t h}$ nearest neighbor is identified in $S$. These points are then used as boundary points of the corresponding $S_{i}$. As a consequence, the partitions of $S$ are overlapping such that for any given point in $R$, all the $k$ nearest neighbors can always be found in a single partition of $S$. An example of $z$-value based partition is given in Figure 2. This method is likely to produce a substantially equivalent number of objects in each partition.

Another similar size based partitioning method can be applied for datasets that are preprocessed with Locality Sensitive Hashing, as illustrated in Figure 3. With this method, the elements of $R$ that are projected to $L S H(R)$, are divided into quasi-equal size partitions using a sampling technique. The same technique can be applied to $S$ and $L S H(S)$.

The strategy of partitioning will impact directly the number of tasks and the amount of computation. Distance based methods aim at dividing the close objects together by preselecting some pivots. Size based methods want to separate objects into equal size zones in which the points are ordered. Regarding the implementation, [25] uses a MapReduce job to

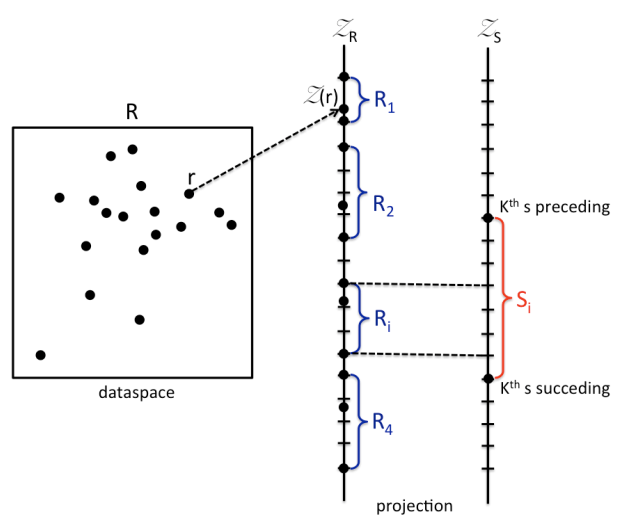

Fig. 2: $z$-value based partitioning

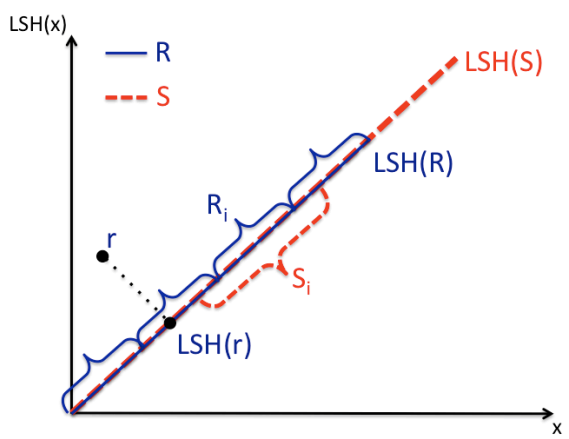

Fig. 3: LSH based partitioning

perform the partitioning. In [26], both data preprocessing and data partitioning are completed in one MapReduce job.

\section{Computation}

The general idea to compute a $\mathrm{kNN}$, is to (i) calculate the distance between $r_{i}$ and $s_{j}$ for all $i$ and all $j$, and (ii) sort these distances in ascending order to find out the first $k$ results. In MapReduce, the idea is the same except that what is given to a task must be independent in order to ensure correctness without duplicating the whole dataset. The number of MapReduce jobs used for computing and sorting has an impact on the global performance, given the complexity of the computation performed by each task and the amount of data to exchange between them. The preprocessing and partitioning steps can also affect the number of tasks that are further needed by each MapReduce job. In this section, we review the different strategies used to compute and sort distances efficiently using MapReduce. These different strategies can be divided into two categories: the ones using one round of MapReduce job and the ones using two rounds of MapReduce jobs. Then, those categories can be divided into two subcategories: the ones that do not preprocess and partition data before computation and the ones that implement the preprocessing and partitioning steps. We detail all these strategies in the following.

1) One Round of MapReduce Job: Without preprocessing and partitioning. The basic idea (H-BkNNJ) to compute a kNN with MapReduce is to have every Map task process a pair of $R_{i}$ and $S_{j}$, and perform a block nested loop on them to calculate the distance between $r_{i} \in R_{i}$ and $s_{j} \in S_{j}, \forall i$ and $j$. Note that, without any smart partitioning strategy, every possible blocks of one partition $R_{i}$ from $R$ and $S_{j}$ from $S$ should be calculated, leading to $n^{2}$ tasks totally where $\mathrm{n}$ is the 


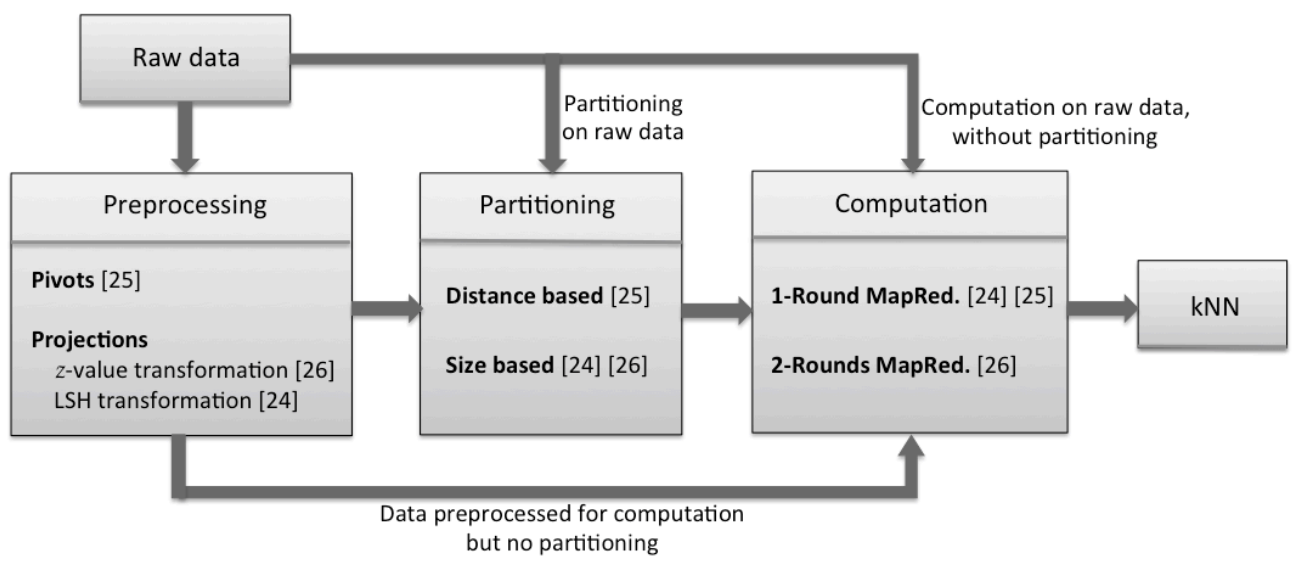

Fig. 4: Usual workflow of a kNN computation using MapReduce

number of partitions of $R$ and $S$. The output of the Map task is in the form of $\left(i d\left(r_{i}\right), \operatorname{list}\left(i d\left(s_{j}\right), d\left(r_{i}, s_{j}\right)\right)\right)$. The identifier of $r_{i}$, named $i d\left(r_{i}\right)$, is used as a key and the associated value is a list containing the identifier of $s_{j}$ and the computed distance between $r_{i}$ and $s_{j}$. A reduce task then processes all computed distances for a given $r_{i}$, and sorts them in ascending order to output the top $k$ results.

With preprocessing and partitioning. To reduce the number of tasks used to calculate the distances, in other words, to reduce the number of pairs formed by $R_{i}$ and $S_{j}$, PGBJ [25] uses a preprocessing and distance based partitioning strategy, which ensures that each partition $R_{i}$ has only one corresponding partition $S_{i}$ needed to be searched, which reduces the number of tasks to $n$. Once the blocks of $R_{i}$ and $S_{i}$ identified, they perform the same calculation as in the H-BkNNJ technique. Because of their data organization, they are able to reduce the number of tasks, greatly improving the performance.

In RankReduce [24] $]^{1}$, the authors first preprocess and partition data into buckets using LSH. Their Map function computes the distance of all points in the partition, and then sorts the local distances to output the local $k$ nearest neighbors for each point in the partition, together with their distance in form of $\left(i d\left(r_{i}\right)\right.$, list $\left.\left(i d\left(s_{j}\right), d\left(r_{i}, s_{j}\right)\right)\right)$. These key-value pairs are then pulled by Reducers where they are sorted and issued as final results.

Overall, the main limitation of these approaches is that the number of values to be sorted in the reduce phase can be extremely large, up to $|S|$, if the pre-processing and partitioning have not significantly reduced the set of candidate points. This greatly limits the applicability of such approach.

2) Two Rounds of MapReduce Jobs: Without preprocessing and partitioning. To overcome the previously described limitation, multiple successive MapReduce jobs are required. The idea is to have the first job output the top $k$ for each pair $\left(R_{i}, S_{j}\right)$. Then, the second job is used to merge all the top $k$ values for a given $r_{i}$ and to perform the merging and sorting of all local top $k$ values (instead of all values), producing the final global top $k$. Such approach is used in H-BNLJ [26] and greatly improves sorting time.

\footnotetext{
${ }^{1}$ Although RankReduce only compute kNN for a single query, it is easy to extend it to a full kNN join
}

With preprocessing and partitioning. A last possibility is based on the z-value preprocessing. In H-zkNNJ [26] the authors propose to generate several shifted copies of $R$ and $S$ (to improve the accuracy), and to determine the bounds of the partitions of $R_{i}$ and the corresponding $S_{i}$ in a preprocessing MapReduce job. So here, the preprocessing and partitioning step is completely integrated in MapReduce. Then, the first MapReduce round of computation takes the partitions $R_{i}$ and $S_{i}$ previously determined, and computes the candidate neighbor set, named $C_{i}(r)$, for $\forall r_{i} \in R_{i}$, which contains $k$ local nearest objects immediately before $r_{i}$ and $k$ objects after $r_{i}$ ( $2 k$ objects totally). The second MapReduce round decides the exact result $\forall r_{i} \in R$ from the candidate neighbor set $k N N\left(r, C_{i}(r)\right)$. So in total, three MapReduce jobs are launched, and among them, two are actually devoted to the $\mathrm{kNN}$ computation. As the number of points that are in the candidate neighbor set is small, thanks to the drastic partitioning (itself due to a drastic preprocessing), the cost of computation and communication is extremely reduced.

\section{Summary}

So far, we have studied the different ways to go through a kNN computation from a workflow point of view with three main steps. The first step focuses on data preprocessing, either for selecting pivots for each partition or for projecting data from high dimension to low dimension. The second step is in charge of data partitioning and organization in the partitions using distance based method or size based method. The last step of the workflow is to actually compute the $\mathrm{kNN}$ in one or two MapReduce jobs. Figure 4 summarizes the workflow we have gone through in this section and the techniques that are associated with each step.

\section{ANALYSIS}

\section{A. Load Balance}

In a MapReduce job, the completion time of the Map phase and the Reduce phase depends on the longest-running task. This makes load balancing particularly important. In this sense, the partitioning strategy should ensure that the processing time of each task is roughly the same, so as to achieve the optimal overall performance. In our context, each task is used to calculate the pairwise distance of $\left\langle r_{i}, s_{i}\right\rangle$ in the partition 


\begin{tabular}{|c|c|c|c|c|c|c|c|}
\hline \multirow[t]{2}{*}{ Systems } & \multirow[t]{2}{*}{ Preprocessing } & \multirow[t]{2}{*}{ Partitioning } & \multirow[t]{2}{*}{ Total jobs } & \multirow[t]{2}{*}{ Accuracy } & \multicolumn{2}{|r|}{ Complexity } & \multirow[t]{2}{*}{ Dynamicity } \\
\hline & & & & & Tasks & Sort & \\
\hline $\begin{array}{l}\text { H-BkNNJ } \\
\text { (basic) }\end{array}$ & None & None & 1 & Exact & $n^{2}$ & $|S| \times \log |S|$ & Static \\
\hline $\begin{array}{l}\text { H-BNLJ [26] } \\
\text { (Zhang et al.) }\end{array}$ & None & Size based & 2 & Exact & $n^{2}$ & $|n \cdot k| \times \log |n \cdot k|$ & Static \\
\hline $\begin{array}{c}\text { RankReduce [24] } \\
\text { (Stupar et al.) }\end{array}$ & LSH & Size based & 2 & Approximate & $n$ & $|n \cdot k| \times \log |n \cdot k|$ & Static \\
\hline $\begin{array}{l}\text { PGBJ [25] } \\
\text { (Lu et al.) }\end{array}$ & $\begin{array}{c}\text { Pivots } \\
\text { selection }\end{array}$ & $\begin{array}{c}\text { Distance based } \\
\text { (Voronoi Diagram) }\end{array}$ & 2 & Exact & $n$ & $\left|S_{i}\right| \times \log \left|S_{i}\right|$ & Static \\
\hline $\begin{array}{l}\text { H-zkNNJ [26] } \\
\text { (Zhang et al.) }\end{array}$ & $Z$-curve & Size based & 3 & Approximate & $n$ & $|2 \cdot k| \times \log |2 \cdot k|$ & Static \\
\hline
\end{tabular}

TABLE I: Summary table of kNN computing systems with MapReduce

$<R_{i}, S_{i}>$. Thus, the number of distances that needs to be calculated is $\left|R_{i}\right| \times\left|S_{i}\right|$. If all tasks have to compute the same number of distances, they should have a roughly equivalent duration. Hence, the optimal partition strategy should make:

$$
\forall \mathrm{i} \neq \mathrm{j},\left|R_{i}\right| \times\left|S_{i}\right|=\left|R_{j}\right| \times\left|S_{j}\right|
$$

This is hard to ensure in practice. It is however possible to obtain a sub-optimal solution in the following situation. Since $S_{i}$ is the set of possible nearest neighbors for the elements in $R_{i}$, then:

$$
\begin{gathered}
\forall \mathrm{i} \neq \mathrm{j}, \\
\text { if }\left|R_{i}\right|=\left|R_{j}\right| \text { or }\left|S_{i}\right|=\left|S_{j}\right|, \\
\text { then }\left|R_{i}\right| \times\left|S_{i}\right| \approx\left|R_{j}\right| \times\left|S_{j}\right|
\end{gathered}
$$

That is to say, if the number of objects in each partition of $R$ is equivalent, then the sum of the number of $k$ nearest neighbors of all objects in each partition can be considered approximately equivalent, and vice versa.

So an efficient partitioning should try to enforce either (1) $\left|R_{i}\right|=\left|R_{j}\right|$ or (2) $\left|S_{i}\right|=\left|S_{j}\right|$.

In [26], the authors give a short proof which shows that the worst-case complexity for (1) is equal to:

$$
O\left(\left|R_{i}\right| \times \log \left|S_{i}\right|\right)=O\left(\frac{|R|}{n} \times \log |S|\right)
$$

and for choice (2), the worst-case complexity is equal to:

$$
O\left(\left|R_{i}\right| \times \log \left|S_{i}\right|\right)=O\left(|R| \times \log \frac{|S|}{n}\right)
$$

where $n$ is the number of partitions. Since $n \ll|S|$, the optimal partitioning is achieved when $\left|R_{i}\right|=\left|R_{j}\right|$.

\section{B. Accuracy}

Usually, the lack of accuracy is the direct consequence of techniques such as $z$-values and LSH used in the preprocessing step. In [26] $(\mathrm{H}-\mathrm{zkNNJ})$, the authors show that when the dimension of the data increases, the quality of the results tends to decrease. This can be counterbalanced by increasing the number of random shifts applied to the data, thereby increasing the size of the resulting dataset. Experiments show that three shifts of the initial dataset (in dimension 15) are sufficient to achieve a good approximation (less than
$10 \%$ of errors shown in the experiences), while controlling the computation time. A similar result can be obtained with LSH by increasing the number of hash functions used. However, when using space filling curves, the distance from the optimal solution (the true $\mathrm{kNN}$ ) is often bounded, which is not the case for LSH, as explained in [30].

\section{Complexity}

Computational complexity is often used to describe the execution time of an algorithm. When computing $\mathrm{kNN}$ with MapReduce, additional factors strongly impact the execution time:

(1) The number of MapReduce jobs: Starting a job (whether in Hadoop [31] or any other platform) requires some initialization steps such as allocating resources and copying data. Those steps can be very time consuming.

(2) The number of Map tasks and Reduce tasks used to calculate $\mathbf{k N N}\left(R_{i} \ltimes S\right)$ : The larger this number is, the more information is exchanged through the network during the Shuffle phase. Moreover, scheduling a task also incurs an overhead.

(3) The number of distances to compute and to sort for each object $r_{i}$ : Sorting is a dominating operation, so the number of elements to be sorted also impacts computation time.

The basic method H-BkNNJ only uses one MapReduce job, and requires $n^{2}$ Map tasks to calculate the distances where $n$ is the number of partitions. The complexity of sorting all distances for one $r_{i}$ in $R$ is $|S| \times \log |S|$. Since $S$ is usually a large dataset, this method quickly becomes impracticable.

To overcome this limitation, H-BNLJ or H-BRJ [26] uses 2 MapReduce jobs, again with $n^{2}$ Map tasks to compute the distances. However, using of a second job significantly reduces the complexity of sorting to $|n \cdot k| \times \log |n \cdot k|$, where $n$ is the number of partitions and $k$ is the number of nearest neighbors queried.

PGBJ [25] performs a pre-processing phase followed by 2 MapReduce jobs. This method also only uses $n$ Map tasks to calculate the distances. Overall, the sorting complexity is reduced to $\left|S_{i}\right| \times \log \left|S_{i}\right|$.

H-zkNNJ [26] also begins by a pre-processing phase and uses in total 3 MapReduce jobs in exchange for taking only $n$ 


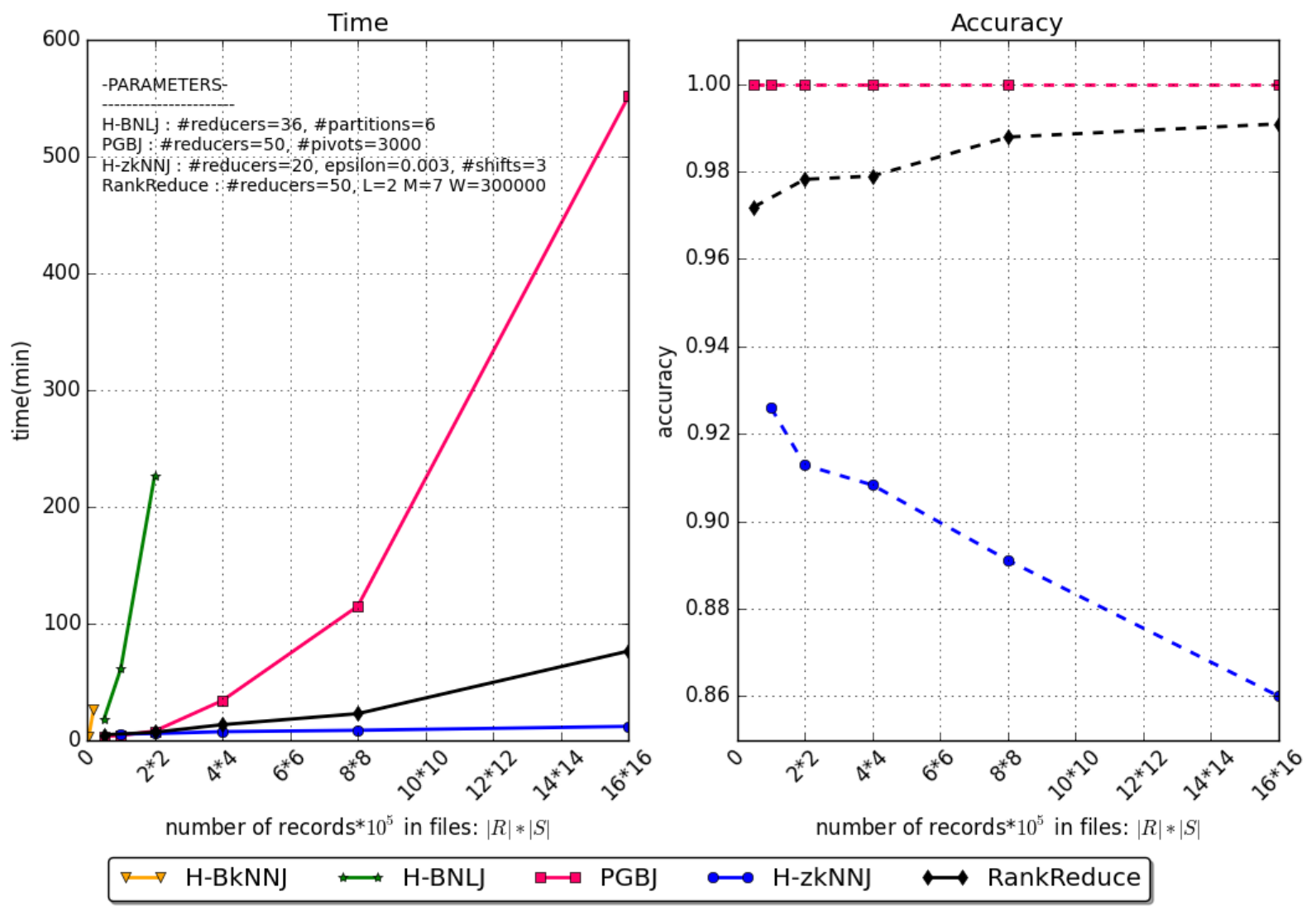

Fig. 5: Completion time and accuracy of knn mapreduce algorithms

Map tasks to compute the distances. Moreover, they only take $\left(r_{i}, C_{i}\left(r_{i}\right)\right)$, that is the candidate neighbors set, into account. Since $C_{i}\left(r_{i}\right)$ contains only $2 k$ neighbors for each $r_{i}$, the complexity is now reduced to $|2 \cdot k| \times \log |2 \cdot k|$.

\section{Wrap up}

Although the workflow for computing $\mathrm{kNN}$ on MapReduce is the same for all existing solutions, the guarantees offered by each of them vary a lot. As load balancing is a key point to shrink completion time, one should carefully choose the partitioning method to achieve this goal. Also, the accuracy of the computing system is crucial: are exact results really needed? If not, then one might trade accuracy for efficiency, by using data transformation techniques before the actual computation. Complexity of the global system should also be taken into account for particular needs, although it is often related to the accuracy: an exact system is usually more complex than an approximate one. Finally, none of the systems really offers a way to handle data updates. This is due to the specific partitioning performed before computing. Indeed, an efficient partitioning is adapted to a particular dataset, and might not be adapted to another. Table I is a summary table of the systems we have examined and their main characteristics.

\section{EXPERIMENTAL EVALUATION}

To validate our analysis, we performed an experimental evaluation of the algorithms we have described in the previous sections. We computed the 20 nearest neighbors (20-NN) of a two dimension geographical dataset on a 20 nodes Hadoop Cluster (dual core with 8 GB of RAM). For each implemented algorithm, we first determined experimentally the optimal parameters (shown in Figure) and show only the best results measured. Figure 5-left shows the completion time of H-BNLJ, RankReduce, PGBJ and H-zkNNJ, for dataset $R$ and $S$ that both went from $0.5 \times 10^{5}$ to $16 \times 10^{5}$ records $(R=S$ at each step). H-BkNNJ could not be executed in reasonable time for big dataset. The execution time of H-BNLJ increases exponentially, making it impracticable for the dataset which contains more than $2 \times 10^{5}$ records. The three other algorithms compete fairly up to the biggest dataset considered, but PGBJ is much slower than the other two. This is expected since it gives an exact result whereas RankReduce and H-zkNNJ are only approximate. Nevertheless, PGBJ greatly improves over the exact algorithm H-BNLJ. Overall, RankReduce and $\mathrm{H}$-zkNNJ are the fastest algorithms to find the approximate nearest neighbors in MapReduce. We also studied their accuracy as shown in Figure 5-right. For small dataset, they both have a good accuracy (97\% for RankReduce vs $93 \%$ for $\mathrm{H}-$ zkNNJ). However, as the dataset grows, the accuracy of $\mathrm{H}-$ zkNNJ decreases to $86 \%$. Because when the dataset grows, the deviation of the estimation will become bigger. But for LSH, when the density of data becomes larger, the chance that the neighbor data being sent to one bucket will increase, that's the reason the accuracy of LSH becomes better when the 
number of data increases. This highlights the trade-off between fastness and accuracy for approximate methods.

\section{CONCLUSION}

In this paper, we have studied the existing systems to perform the $\mathrm{kNN}$ operation in the context of MapReduce. We have first approached this problem from a workflow point of view. We have pointed out that all solutions follow three main steps to compute kNN over MapReduce, namely the pre-processing of data, the partitioning and the actual computation. We have listed and explained the different algorithms which could be chosen for each step, and developed their pros and cons. In a second stage, we have further analyzed existing systems by reviewing their main properties, in terms of load balancing, accuracy of the computation, and overall complexity. Above all, this paper can be seen as a guideline to help selecting the most appropriate method to perform the $\mathrm{kNN}$ join operation on MapReduce for a particular use case.

\section{ACKNOWLEDGMENT}

The authors would like to thank Léa El Beze for her work on the experimentation. Experiments presented in this paper were carried out using the Grid' 5000 testbed, supported by a scientific interest group hosted by Inria and including CNRS, RENATER and several Universities as well as other organizations (see https://www.grid5000.fr)

\section{REFERENCES}

[1] D. Li, Q. Chen, and C.-K. Tang, "Motion-aware knn laplacian for video matting," in International Conference on Computer Vision (ICCV), 2013, 2013.

[2] H.-P. Kriegel and T. Seidl, "Approximation-based similarity search for 3-d surface segments," Geoinformatica, vol. 2, no. 2, pp. 113-147, Jun. 1998

[3] X. Bai, R. Guerraoui, A.-M. Kermarrec, and V. Leroy, "Collaborative personalized top-k processing," ACM Trans. Database Syst., vol. 36, no. 4, pp. 26:1-26:38, Dec. 2011.

[4] D. Rafiei and A. Mendelzon, "Similarity-based queries for time series data," SIGMOD Rec., vol. 26, no. 2, pp. 13-25, Jun. 1997

[5] R. Agrawal, C. Faloutsos, and A. N. Swami, "Efficient similarity search in sequence databases," in Proceedings of the 4th International Conference on Foundations of Data Organization and Algorithms, ser FODO '93. London, UK, UK: Springer-Verlag, 1993, pp. 69-84.

[6] K. Inthajak, C. Duanggate, B. Uyyanonvara, S. Makhanov, and S. Barman, "Medical image blob detection with feature stability and knn classification," in Computer Science and Software Engineering (JCSSE), 2011 Eighth International Joint Conference on, May 2011, pp. 128-131.

[7] F. Korn, N. Sidiropoulos, C. Faloutsos, E. Siegel, and Z. Protopapas, "Fast nearest neighbor search in medical image databases," in Proceedings of the 22th International Conference on Very Large Data Bases, ser. VLDB '96. San Francisco, CA, USA: Morgan Kaufmann Publishers Inc., 1996, pp. 215-226.

[8] H. V. Jagadish, B. C. Ooi, K.-L. Tan, C. Yu, and R. Zhang, "idistance: An adaptive $\mathrm{b}^{+}$-tree based indexing method for nearest neighbor search," ACM Trans. Database Syst., vol. 30, no. 2, pp. 364-397, 2005.

[9] C. Böhm and F. Krebs, "The k-nearest neighbour join: Turbo charging the kdd process," Knowl. Inf. Syst., vol. 6, no. 6, pp. 728-749, Nov. 2004.

[10] P. Ciaccia, M. Patella, and P. Zezula, "M-tree: An efficient access method for similarity search in metric spaces," in Proceedings of the 23rd International Conference on Very Large Data Bases, ser. VLDB '97. San Francisco, CA, USA: Morgan Kaufmann Publishers Inc. 1997, pp. 426-435.
[11] C. Yu, R. Zhang, Y. Huang, and H. Xiong, "High-dimensional knn joins with incremental updates," GeoInformatica, vol. 14, no. 1, pp. 55-82, 2010.

[12] J. L. Bentley, "Multidimensional binary search trees used for associative searching," Commun. ACM, vol. 18, no. 9, pp. 509-517, Sep. 1975.

[13] J. Dean and S. Ghemawat, "Mapreduce: Simplified data processing on large clusters," Commun. ACM, vol. 51, no. 1, pp. 107-113, Jan. 2008.

[14] “Hadoop." [Online]. Available: http://hadoop.apache.org/

[15] N. Bhatia and A. Vandana, "Survey of nearest neighbor techniques," International Journal of Computer Science and Information Security, vol. 8, no. 2, 2010.

[16] L. Jiang, Z. Cai, D. Wang, and S. Jiang, "Survey of improving k-nearestneighbor for classification," in Proceedings of the Fourth International Conference on Fuzzy Systems and Knowledge Discovery - Volume 01, ser. FSKD '07. Washington, DC, USA: IEEE Computer Society, 2007, pp. 679-683.

[17] C. Yu, R. Zhang, Y. Huang, and H. Xiong, "High-dimensional knn joins with incremental updates," Geoinformatica, vol. 14, no. 1, pp. 55-82, Jan. 2010.

[18] M. I. Andreica and N. T. Ăpus, "Sequential and mapreduce-based algorithms for constructing an in-place multidimensional quad-tree index for answering fixed-radius nearest neighbor queries," 2013.

[19] M. Datar, N. Immorlica, P. Indyk, and V. S. Mirrokni, "Localitysensitive hashing scheme based on p-stable distributions," in Proceedings of the Twentieth Annual Symposium on Computational Geometry, ser. SCG '04. New York, NY, USA: ACM, 2004, pp. 253-262.

[20] B. Yao, F. Li, and P. Kumar, "K nearest neighbor queries and knn-joins in large relational databases (almost) for free," in Data Engineering (ICDE), 2010 IEEE 26th International Conference on, March 2010, pp. $4-15$.

[21] A. S. Arefin, C. Riveros, R. Berretta, and P. Moscato, "Gpu-fs-knn: A software tool for fast and scalable knn computation using gpus," PLOS ONE, vol. 7, no. 8, Aug. 2012.

[22] D. Novak and P. Zezula, "M-chord: A scalable distributed similarity search structure," in Proceedings of the 1st International Conference on Scalable Information Systems, ser. InfoScale '06. New York, NY, USA: ACM, 2006

[23] P. Haghani, S. Michel, and K. Aberer, "Lsh at large - distributed knn search in high dimensions ," 2008.

[24] A. Stupar, S. Michel, and R. Schenkel, "Rankreduce - processing knearest neighbor queries on top of mapreduce," in In LSDS-IR, 2010.

[25] W. Lu, Y. Shen, S. Chen, and B. C. Ooi, "Efficient processing of k nearest neighbor joins using mapreduce," Proc. VLDB Endow., vol. 5, no. 10, pp. 1016-1027, Jun. 2012.

[26] C. Zhang, F. Li, and J. Jestes, "Efficient parallel knn joins for large data in mapreduce," in Proceedings of the 15th International Conference on Extending Database Technology, ser. EDBT '12. New York, NY, USA: ACM, 2012, pp. 38-49.

[27] M. Bawa, T. Condie, and P. Ganesan, "Lsh forest: Self-tuning indexes for similarity search," in Proceedings of the 14th International Conference on World Wide Web, ser. WWW '05. New York, NY, USA: ACM, 2005, pp. 651-660.

[28] G. Song, Z. Meng, F. Huet, F. Magoulès, L. Yu, and X. Lin, "A hadoop mapreduce performance prediction method," in 10th IEEE International Conference on High Performance Computing and Communications \& 2013 IEEE International Conference on Embedded and Ubiquitous Computing, HPCC/EUC 2013, Zhangjiajie, China, November 13-15, 2013, 2013, pp. 820-825.

[29] X. Zhou, D. J. Abel, and D. Truffet, "Data partitioning for parallel spatial join processing," Geoinformatica, vol. 2, no. 2, pp. 175-204, Jun. 1998.

[30] S. Liao, M. A. Lopez, and S. T. Leutenegger, "High dimensional similarity search with space filling curves," in Data Engineering. IEEE, 2001, pp. 615-622.

[31] D. Jiang, B. C. Ooi, L. Shi, and S. Wu, "The performance of mapreduce: An in-depth study," Proc. VLDB Endow., vol. 3, no. 1-2, pp. 472-483, Sep. 2010. 